\title{
„Ich lass mich da nicht klein machen!“‘ Eine qualitative Studie zur Bewältigung von Hatespeech durch Schüler/innen
}

\author{
Norman Krause $\mathbb{D} \cdot$ Cindy Ballaschk • Friederike Schulze-Reichelt • \\ Julia Kansok-Dusche • Sebastian Wachs • Wilfried Schubarth • \\ Ludwig Bilz
}

Eingegangen: 30. September 2020 / Überarbeitet: 4. März 2021 / Angenommen: 9. März 2021 / Online publiziert: 4. Mai 2021

(C) Der/die Autor(en) 2021

Zusammenfassung Von Hass und Hetze geprägte Kommunikationsformen im Internet stehen seit einigen Jahren im Fokus der öffentlichen Aufmerksamkeit. Kaum beachtet wurden bisher jedoch die Fragen, ob sich solche als Hatespeech bezeichneten Ausdrucksweisen auch in der Schule zeigen und wie Schüler/innen derartige Vorfälle bewerten und bewältigen. Um Antworten auf diese Fragen zu finden, wurden 21 leitfadengestützte, episodische Interviews (nach Flick) mit Jugendlichen aus Berlin und Brandenburg zu ihren Erfahrungen und Bewältigungsstrategien in Bezug auf Hatespeech im schulischen Kontext ausgewertet. Die Ergebnisse machen deutlich, dass Hatespeech-Erfahrungen von Schüler/innen als belastend wahrgenommen und die eigenen Potenziale zur Bewältigung individuell und situativ unterschiedlich bewertet werden. Insbesondere die subjektive Beeinflussbarkeit bzw. Kontrollierbarkeit des stressauslösenden Hatespeech-Vorfalls sowie die soziale Unterstützung im Setting der Schulklasse scheinen sich auf die Bewältigung auszuwirken. Die berichteten Bewältigungsstrategien lassen sich, dem Transaktionalen Stressmodell entsprechend, in emotionsfokussiert (Einbezug sozialer Unterstützung, Verdrängung und Vermeidung) und problemfokussiert (aktives Ignorieren und Counterspeech) kategorisieren. Die Ergebnisse stützen Forderungen nach Präventionsarbeit in Schulen. Schüler/innen sollten dabei als Expert/innen der Lebenswelt Schule einbezogen werden. Außerdem scheinen Maßnahmen zur Stärkung sozialer und personaler Ressourcen sinnvoll zu sein, um konstruktive Bewältigungsstrategien zu ermöglichen.

\footnotetext{
N. Krause $(\varangle) \cdot$ C. Ballaschk $\cdot$ F. Schulze-Reichelt $\cdot$ S. Wachs $\cdot$ W. Schubarth Department Erziehungswissenschaft, Universität Potsdam, Karl-Liebknecht-Str. 24-25, 14476 Potsdam, Deutschland

E-Mail: normankrause@uni-potsdam.de

J. Kansok-Dusche $\cdot$ L. Bilz

Fakultät für Soziale Arbeit, Gesundheit und Musik, Brandenburgische Technische Universität Cottbus-Senftenberg, Universitätsplatz 1, 01968 Senftenberg, Deutschland
} 
Zur Konkretisierung und Quantifizierung der Forschungsergebnisse ist weitere Forschung notwendig.

Schlüsselwörter Hatespeech $\cdot$ Hassrede $\cdot$ Bewältigung $\cdot$ Schule $\cdot$ Diskriminierung

\title{
"I don't let them get me down!"-A qualitative study on students' coping with hate speech
}

\begin{abstract}
Forms of communication on the Internet characterized by hatred and agitation have been in the focus of public attention since several years. The questions whether such expressions (known as hate speech) are also noticeable in schools and how students evaluate and cope with such incidents still remain unanswered. In order to find answers to these questions, 21 guideline-based, episodic interviews (according to Flick) with German students from Berlin and Brandenburg on experiences and coping strategies regarding to hatred in school contexts were evaluated. As the results show, students perceive hate speech experiences as stressful. They assess their own coping resources individually and situationally different. In particular, the subjectively perceived influenceability or controllability of the stress-inducing hate speech incident and the social support within the school class seem to have effects on coping. The reported coping strategies can be categorized in accordance to the transactional model of stress into emotion-focused (reference to social support, repression and avoidance) and problem-focused (active ignoring and counter speech). The results support demands for prevention work in schools. Students should be involved as experts of the school environment. Furthermore, actions to strengthen social and personal resources seem to be useful in order to enable constructive coping strategies. More research is necessary to define and quantify the research results.
\end{abstract}

Keywords Hate speech $\cdot$ Counter speech $\cdot$ Coping $\cdot$ School $\cdot$ Discrimination

\section{Einleitung}

Menschenverachtende Posts, Kommentare, Bilder oder Videos gegen Personengruppen (z. B. Geflüchtete, Homosexuelle, Muslime) sind in sozialen Medien stark verbreitet (Hawdon et al. 2019). Ein Fachbegriff für eine solche kommunikative Abwertung und Verachtung von Personengruppen ist Hatespeech (Wachs et al. 2020a). Wenn man Jugendliche allerdings fragt, wo sie mit Hatespeech am häufigsten konfrontiert werden, wird nach dem Internet am häufigsten die Schule genannt (UK Safer Internet Centre 2016). Bisher gibt es zu Hatespeech in der Schule jedoch kaum Forschung.

Von Hatespeech betroffen zu sein, stellt u. a. eine Gewalterfahrung, einen Angriff auf die soziale Identität und eine Verletzung der Menschenwürde dar (Wachs et al. im Druck). Deshalb verwundert es nicht, dass Hatespeech-Erfahrungen - ähnlich wie Erfahrungen mit Diskriminierungen - mit negativen Folgen für das Wohlbefinden einhergehen (Hansen 2009; Gámez-Guadix et al. 2020). Um besser zu verstehen, 
wie sich diese Folgen vermeiden lassen und wie solche Erfahrungen bewältigt werden können, bedarf es empirischer Erkenntnisse. Bisher liegt im deutschsprachigen Raum jedoch kaum empirisch fundiertes Wissen darüber vor, welche Strategien Jugendliche nutzen, um Diskriminierung im Allgemeinen (Scherr und Breit 2018) und ganz speziell Hatespeech in der Schule zu bewältigen, und welche Unterstützung sie sich wünschen. Hier setzt der vorliegende Beitrag an, indem erstmals episodische Interviews mit Jugendlichen dazu geführt werden, wie sie Hatespeech-Viktimisierung in der Schule erleben und bewältigen. Durch das qualitative, weitgehend induktivmethodische Vorgehen werden die Schüler/innen in besonders offener Weise als Akteur/innen der Schule berücksichtigt. Aus ihren Aussagen können Bewältigungsstrategien spezifisch für Hatespeech in der Schule entnommen und entsprechende Potenziale erkannt werden. Die Ergebnisse können als Grundlage für weiterführende Forschung und für die Entwicklung schulischer Präventionsprogramme genutzt werden.

\section{Hintergrund der Studie}

\subsection{Bewältigung von Hatespeech aus transaktionaler Perspektive}

Werden Personen wegen ihrer Zugehörigkeit zu einer Gruppe verunglimpft, beleidigt oder abgewertet, kann dies als stressvoll und belastend empfunden werden. Die Auswirkungen für die Betroffenen können sich u. a. in negativen Emotionen (z. B. Scham, Trauer, Wut), reaktiven Aggressionen, einem geringen Selbstwertgefühl, geringem Wohlbefinden oder Desintegrationstendenzen zeigen (Chavez et al. 2019; Eisenberg et al. 2003; Gámez-Guadix et al. 2020; Goffman [1963] 2012; Saha et al. 2019; Soral et al. 2018; Tynes et al. 2008; UK Safer Internet Centre 2016; Wright und Wachs 2019). Um mögliche Folgen von Hatespeech besser zu verstehen, können Stressmodelle herangezogen werden, die Aufschluss darüber geben, wie Betroffene Hatespeech-Erfahrungen bewältigen.

Das am häufigsten untersuchte Modell zur Bewältigung von Stress ist das Transaktionale Stressmodell (Lazarus und Folkman 1984). Aus dieser Perspektive entsteht Stress aus einem Ungleichgewicht zwischen Anforderungen aus der Umwelt und den individuellen Möglichkeiten und Ressourcen, mit den Anforderungen zurecht zu kommen (Lazarus und Folkman 1984). Der Prozess der Bewältigung umfasst kognitive Bewertungen und Bewältigungsstrategien. Die Evaluation des Stressors (z.B. Hatespeech-Übergriff) erfolgt in drei Schritten. In einem ersten Schritt (primäre Bewertung) wird die Situation vom Individuum subjektiv hinsichtlich ihrer Folgen interpretiert, d.h. sind diese positiv, irrelevant oder negativ. Bei negativen Folgen wird weiter differenziert, ob der Stressor z.B. als Herausforderung oder Bedrohung wahrgenommen wird. In einem zweiten Schritt werden vorhandene Bewältigungspotenziale analysiert (sekundäre Bewertung). Dabei werden eigenes Verschulden, situationsbedingte Handlungsoptionen bzw. bekannte Strategien und vorhandene Ressourcen abgewogen. In Abhängigkeit dessen werden Bewältigungsstrategien aktiviert, die sich in zwei Kategorien unterscheiden lassen (Lazarus und Folkman 1984): Die problemorientierten Bewältigungsstrategien, bei denen das In- 
dividuum ereignis- bzw. situationsbezogen handelt, um den Stressor zu verändern oder zu beseitigen, und die emotionsorientierten Bewältigungsstrategien, bei der durch Emotionsregulation die Bewertung des Stressors angepasst wird, nicht jedoch das Ereignis selbst. Problemorientierte Strategien werden eher angewendet, wenn der Stressor als kontrollierbar bzw. beeinflussbar wahrgenommen wird, während bei weniger kontrollierbaren Stressoren emotionsorientierte Strategien der Anpassung und Vermeidung bevorzugt werden (Compas et al. 2001). In einem letzten Schritt können Neubewertungen auf Grundlage von Veränderungen oder Rückmeldungen bzw. Ergebnissen aus der Transaktion zwischen Individuum und Umwelt vorgenommen werden (Lazarus und Folkman 1984).

Bisher liegen lediglich erste Befunde dazu vor, wie Jugendliche Hatespeech im Internet bewältigen. In einer Befragung britischer Jugendlicher zwischen 13 und 18 Jahren gaben die meisten an, Hatespeech zu ignorieren, gefolgt davon, den Inhalt der Plattform zu melden, mit einer/einem Freund/in darüber zu sprechen, die Person zu blockieren, die Hatespeech geäußert hat, einer/einem Erwachsenen davon zu berichten, mit Counterspeech zu reagieren, also die Person online zur Rede zu stellen und eine Lehrkraft oder die Polizei zu informieren (UK Safer Internet Centre 2016). Dieselbe Studie zeigt, dass mehr als ein Drittel der Befragten Hatespeech als besorgniserregende Entwicklung bewertet und mit Hatespeech negative Emotionen verbunden sind. In einer aktuelleren Studie mit deutschen Heranwachsenden im Alter von 12 bis 17 Jahren konnten Wachs et al. (2020) zeigen, dass Jugendliche zwar überwiegend konstruktive Bewältigungsstrategien nutzen (z. B. technische Bewältigung durch Blocken und Melden, Suchen nach sozialer Unterstützung oder Counterspeech), doch etwa ein Viertel sich im Umgang mit Hatespeech hilflos fühlt und etwa ein Fünftel Rache als mögliche Reaktion angibt. Daran anschließende Untersuchungen zeigten u.a., dass Opfer von Online-Hatespeech seltener selbstbehauptend reagieren oder technologische Bewältigung anwenden und häufiger Rache als geeignete Reaktion auf Online-Hatespeech betrachten (Wachs et al. 2020b). Bewältigungsstrategien sind auch deshalb als bedeutsam zu betrachten, weil erste Hinweise vorliegen, dass Hilflosigkeit im Umgang mit Online-Hatespeech negativ und Bewältigungsstrategien wie z.B. soziale Unterstützung oder Selbstbehauptung positiv mit Wohlbefinden im Jugendalter zusammenhängen (Gámez-Guadix et al. 2020).

\subsection{Forschungsfragen}

Vor dem Hintergrund der beschriebenen Forschungslücken, lassen sich folgende Forschungsfragen formulieren:

1. Wie bewerten Schüler/innen gemäß des Transaktionalen Stressmodells die Stressrelevanz von Hatespeech und die eigenen Bewältigungspotenziale im Umgang damit?

2. Welche Bewältigungsstrategien berichten Jugendliche hinsichtlich ihres Umgangs mit Hatespeech im Schulkontext und welche Auswirkungen haben Bewältigungserfahrungen auf den zukünftigen Umgang mit Hatespeech? 


\section{Methode}

\subsection{Sampling und Feldzugang}

Die Stichprobe besteht aus 55 geschlechtlich unterschiedlich positionierten sowie unterschiedlich ethnisierten Zehntklässler/innen von sieben Schulen aus Berlin und Brandenburg. Eine induktive Stichprobenziehung bzw. ein theoretisches Sampling (nach Grounded Theory), bei dem die Fallauswahl im laufenden Prozess und auf der Basis erster Ergebnisse zirkulär angepasst werden kann (Glaser und Strauss 1998 [1967]), ist aufgrund der landesbehördlichen Genehmigungsverfahren nicht möglich, da die Fallauswahl bereits vor der Erhebung feststehen musste. Deshalb wurde kriteriengeleitet vorab eine möglichst kontrastreiche Fallauswahl getroffen (,,purposeful Sampling“) (Palinkas et al. 2015). Kontrastiert wurde durch die Erhebung in Berlin und Brandenburg, wodurch eine Unterscheidung zwischen ländlichen und (groß-)städtischen Gebieten abgebildet ist, sowie innerhalb Brandenburgs zwischen größeren Städten und kleinstädtischer oder ländlicher Lage und innerhalb Berlins, abhängig von der Lage der Schule, nach sozioökonomischem Milieu. Zudem wurden Schulen aus West- und Ostberlin erhoben. Unterschieden wurde auch nach Schulform, insbesondere zwischen dem Gymnasium sowie der Integrierten Sekundarschule (Berlin) bzw. der Oberschule (Brandenburg). Darunter gibt es sowohl solche Schulen, die über eine gymnasiale Oberstufe verfügen, als auch solche, die dieses Angebot nicht haben, woraus sich Unterschiede unter den Schüler/innen hinsichtlich des angestrebten Bildungsabschlusses ergeben.

Der Feldzugang erfolgte über eigene Netzwerke, Gatekeeper (z. B. Gewerkschaften, Verbände, Parteien) und direkte Anfragen bei den Schulen. Die Teilnahme an der Studie basierte auf Freiwilligkeit. Alle Teilnehmenden wurden vor den Interviews über den Zweck der Erhebung, die anonymisierte Datenauswertung und ihre Rechte informiert. Vor Durchführung der Studie wurden ein Ethikvotum (UP65/2018) und die Genehmigungen der Landesbehörden eingeholt.

\subsection{Untersuchungsinstrumente}

Die Datenerhebung an den Schulen erfolgte mittels teilstrukturierendem Leitfaden. Dieser beinhaltete u. a. Fragen und Erzählaufforderungen zu verschiedenen Formen und Arten von Hatespeech sowie zu Strategien, die Jugendliche im Umgang mit Hatespeech nutzen. Durch eine Kombination von offenen Erzählaufforderungen zu (schul-)biografischem, erfahrungsnahem und situationsbezogenem Wissen und konkreten, zielgerichteten Fragen zu semantischem Wissen entspricht dies nach Flick (2019) einem episodischen Interview. Die Fragen und Erzählaufforderungen wurden mehrfach unter dem Anspruch der maximalen Offenheit reflektiert und entsprechend modifiziert (Helfferich 2014), sodass die Interviewten ihre subjektive Sicht möglichst unbeeinflusst entfalten können. In den Interviews wurde zudem ein Schaubild genutzt, um das Phänomen Hatespeech zu erläutern und gegenüber anderen Phänomenen abzugrenzen. Dieses wurde in der Regel nach den Fragen zum subjektiven (Alltags-)Verständnis und entsprechenden Beispielen präsentiert. 


\subsection{Datenauswertung und -analyse}

Aus der Gesamtzahl der Interviews wurde aufgrund der großen Menge kriteriengeleitet eine Materialauswahl von 21 Interviews zur weiteren Auswertung getroffen (J01-J21). Es wurden sukzessive solche Interviews ausgewählt, die neue relevante Aspekte, z. B. noch nicht berichtete Strategien im Umgang mit Hatespeech, oder besonders ergiebige biografische Erzählungen enthielten. Diese wurden angelehnt an das Transkriptionssystem GAT2 (Dresing und Pehl 2018) softwaregestützt verschriftlicht. Die Transkripte enthalten den exakten Wortlaut der Redebeiträge sowie Informationen zur Verlaufsstruktur. Personenbezogene Daten wurden während der Verschriftlichung anonymisiert oder entfernt.

Dem Leitfaden zugrundeliegende Erkenntnisinteressen wurden, am inhaltsanalytischen Vorgehen orientiert (Mayring 2015), auf das Kategoriensystem deduziert, das dementsprechend vorgegliedert wurde. Die weitere Datenauswertung orientierte sich am Vorgehen nach der Grounded Theory (Strauss und Corbin 2010; Strübing 2014) in dem Sinne, dass ein entsprechend induktives Analyseverfahren verfolgt wurde: Aus den Transkripten wurden innerhalb der bestehenden Kategorien induktiv, zunächst sogar „in-vivo“, Zitate codiert (offenes Codieren) und daraus im weiteren Verlauf neue Kategorien generiert bzw. nach Diskussion im Forschungsteam, Anpassungen des Kategoriensystems vorgenommen (axiales und selektives Codieren). Mithilfe des verwendeten Programms MAXQDA wurden zudem softwaregestützt Code- und Dokumentenmemos erstellt. Diese enthalten Interpretationen und Hypothesen der Forschenden und unterstützen die Theoriebildung im Sinne der Grounded Theory.

Die Ergebnisse der Analyse wurden anschließend diskursiv im Forschungsteam mit bestehenden Theorien und Modellen zur Bewältigung von Belastungserleben in Verbindung gebracht. Hierbei war die Konvergenz zwischen dem Datenmaterial und dem Transaktionalen Stressmodell besonders hoch. Das Transaktionale Stressmodell berücksichtigt neben Reaktionen auch Wahrnehmungs- und Bewertungsprozesse, die sich auch in unserem Datenmaterial zeigten. Zudem wurde es bereits im Hinblick auf verwandte Phänomene, wie Mobbing und Cyberhate, erfolgreich angewendet (Hunter und Boyle 2004; Wright et al. 2021).

\subsection{Datengüte}

Zur Sicherung der Datengüte wurde die prozedurale Reliabilität vorab insofern beachtet, als dass die Datenaufzeichnung standardisiert erfolgte (Tonbandaufnahme und standardisierte Verschriftlichung) (Flick 2014). Weiterhin wurde Wert auf die Fallkontrastierung gelegt. Zudem wurden verschiedene Formen der Triangulation (Denzin 1989; Flick 2019) beachtet: Es wurden, entsprechend der Kontrastierung im Sampling, systematisch unterschiedliche Schulen einbezogen und verschiedene Personen (an mehreren Orten und Zeitpunkten) interviewt (Daten-Triangulation). Verzerrungen durch die Person des Forschenden konnten minimiert werden, indem Datenerhebung und -auswertung von verschiedenen Personen durchgeführt, diese dafür sensibilisiert und die Arbeitsprozesse und die eigene Subjektivität im Team reflektiert wurden (Forschenden-Triangulation). Veränderungen am Kategoriensys- 
tem wurden nach diskursiver Einigung der an der Auswertung beteiligten Forschenden vorgenommen. Verzerrungen durch vorgefasste Denkmuster und Vorstellungen konnten durch die Einbeziehung verschiedener Hypothesen und Perspektiven aufgedeckt und verringert werden (Theorien-Triangulation). Durch die Form des episodischen Interviews konnte durch die Kombination von Erzählaufforderungen (Erfahrungs- und Biografiewissen) und Nachfragen (semantisches Wissen), ,withinmethod" trianguliert werden (Methodologische Triangulation).

\section{Ergebnisse}

Die befragten Jugendlichen berichten aus Betroffenen- und Beobachterperspektive von subtilen bis hin zu sehr offenen Hatespeech-Erfahrungen in der Schule. Diese werden z. B. als rassistisch (z. B. aufgrund der Hautfarbe oder Herkunft), sexistisch, homo- und transfeindlich, gegen die Religion (z.B. antisemitisch, antimuslimisch) oder das äußerliche Erscheinungsbild gerichtet beschrieben. Zu bewältigende Vorfälle sind überwiegend aus der Betroffenenperspektive geschildert. Die Präsentation der folgenden Ergebnisse orientiert sich strukturell am Transaktionalen Stressmodell. So werden zunächst primäre und sekundäre Bewertungsprozesse beschrieben, anschließend Bewältigungsstrategien und zuletzt Hinweise auf eine Neubewertung.

\subsection{Primäre und sekundäre Bewertung}

Mehrere Passagen deuten darauf hin, dass Hatespeech-Viktimisierung durch Jugendliche als stressrelevant erlebt wird (primäre Bewertung). Entweder berichten Jugendliche direkt von den mit Hatespeech verbundenen Belastungen (z. B. „Das ist ja wirklich etwas, was einen tief bedrückt“ (J19)) oder indirekt durch Berichte negativer Emotionen als Folge von Hatespeech-Viktimisierung, die als Belastungserleben interpretiert werden können. So wurde z.B. von Angst berichtet: „Also ich hatte echt Angst, so durch die Schule zu gehen“ (J03). Auch Wut als Folge von Hatespeech-Viktimisierung, die in reaktive Aggressionen mündete, wurde beschrieben: ,[Dann] hatte ich Hass in mir und dieser Hass wurde dann [...] ausgenutzt, um andere Menschen zu verletzen“" (J11).

Den Interviews lassen sich zudem Bewertungen der eigenen und allgemeinen Bewältigungsfähigkeit sowie mangelnder und vorhandener bewältigungsdienlicher Ressourcen entnehmen (sekundäre Bewertung). Eine gering bewertete Bewältigungsfähigkeit wurde berichtet, wenn Schüler/innen keine Handlungsoptionen erkannten, z. B.: ,[...] man kann wirklich nicht viel [da]gegen machen, außer zu hoffen, dass diese Menschen es einfach kapieren, dass es nichts Gutes ist" (J19). Weiterhin wurde das entwicklungsbedingte Fehlen von geeigneten Bewältigungsstrategien genannt: „Ich glaube, viele junge Leute können mit solchen Beleidigungen [...] nicht umgehen, weil sie noch nicht wissen, wie“ (J02) oder „Aber andere Leute, die damit nicht umgehen können, brechen halt dann zusammen“ (J18). Dagegen lassen sich auch Hinweise auf eine subjektiv hoch bewertete Bewältigungsfähigkeit im Datenmaterial finden: „Ich bin die Einzige, die daran was ändern könnte, die anderen können das ja nicht ändern“"(J02). 


\subsection{Bewältigungsstrategien im Umgang mit Hatespeech}

\subsubsection{Emotionsfokussierte Bewältigungsstrategien}

Emotionsfokussierte Bewältigungsstrategien zielen darauf ab, emotionale Erregung, die durch den Stressor entsteht, abzubauen. Eine zentrale Form emotionsfokussierter Bewältigung, die sich im Datenmaterial entdecken lässt, stellt der Einbezug sozialer Unterstützung dar, z. B.: ,[...] solange du deine Freunde hast und du dich auf deine Freunde verlassen kannst, dann ist es egal, was andere über dich sagen“ (J12). Auch in einem anderen Interview wird auf positive Beziehungen zu Peers als wichtige Bewältigungsstrategie hingewiesen: „Also mir reicht's, wenn mir jemand sagt, ich bin für dich da, weil das ziemlich schwer ist, wenn wirklich nur alle gegen einen sind. Und da reicht's dann schon [...], dass derjenige [...] einfach sagt, ich versteh, was du meinst, ich steh hinter dir. Das gibt 'n [...] gutes Gefühl und das sorgt auch dafür, dass man sich eben nicht alleine fühlt in dieser Welt" (J05). Solche Aussagen deuten darauf hin, dass soziale Unterstützung durch Peers emotionale Stabilität bieten kann und zentral ist, um negative Emotionen durch Hatespeech-Übergriffe zu bewältigen. An anderen Stellen wird zudem der Zusammenhang zwischen sozialer Unterstützung und Bewältigungsfähigkeit hergestellt, z.B.: „wenn du keine Lehrer hast, [...] die dich da so unterstützen, dann hast du ja keine andere Wahl als dann einfach die Zähne zusammen zu beißen“" (J01). Bedenklich ist, dass eine stabile soziale Eingebundenheit umgekehrt dazu führen kann, dass hemmende soziale Mechanismen bezüglich Hatespeech fehlen. J18 äußert dazu über Hatespeech ausübende Personen: „Und wenn das bei ihnen selbst passieren würde, würden sie sich halt einfach in Gruppen schließen und dann auf diese Person, die denjenigen runtergemacht hat, [...] drauf gehen" (J18).

Eine weitere Form des emotionsfokussierten Umgangs, die sich im Datenmaterial finden lässt, ist Verdrängung. Hierbei soll die belastende Situation aus dem Bewusstsein verschoben oder vergessen werden: „Okay, also ich versuche das einfach zu vergessen. [...] klappt nicht immer, gerade wenn [...] man die Personen ständig sieht““ (J01). Diese Strategie ist, wie in dieser Aussage deutlich wird, in der Schule deutlich limitierter als im Internet. Bei erhöhter Wiederholungswahrscheinlichkeit ist sie als wenig funktional einzuschätzen.

Auch aktive Vermeidung kann als emotionsfokussierte Strategie gelten, denn so sollen Ängste durch Vermeidung des Stressors reduziert werden. Vermeidende Strategien zeigten sich, indem gewisse Orte, Personen oder Situationen gemieden wurden (,Wenn man nicht den Mut hat [...] sich mit dem zu streiten, einfach weggehen“ (J20)), aber auch in Extremhandlungen wie z. B. Schuldistanz oder gar Schulwechsel: „Und wir hatten auch zweimal Muslime in der Klasse. Aber die kamen dann halt einfach nicht zur Schule, weil sie ausgegrenzt werden und das nach einer Woche einfach nicht mehr aushalten" (J18).

\subsubsection{Problemfokussierte Bewältigungsstrategien}

Problemfokussierte Bewältigungsstrategien haben das Ziel, den Stressor selbst zu ändern. Eine solche Strategie stellt Counterspeech (Gegenrede) dar. Sie umfasst 
Möglichkeiten, um auf Hatespeech kommunikativ und in Auseinandersetzung mit der Person, die die Hatespeech geäußert hat, zu reagieren. Ziel der Counterspeech ist eine konstruktive Aufarbeitung zur Beendigung der Hatespeech. Zur Kategorie Counterspeech konnten unterschiedliche Handlungen zugeordnet werden: Zunächst ist das Positionieren eine Form der Counterspeech. Vordergründig ist, der Hatespeech etwas entgegenzusetzen und sie nicht unwidersprochen stehen zu lassen, üblicherweise ebenfalls öffentlich und mit Botschaftscharakter. Mitunter geht das mit Empörung einher: ,[...] wenn ich eben betroffen bin, lass ich mich da nicht klein machen. Also, wenn's berechtigte Kritik ist, dann nehme ich mir die zu Herzen, aber nicht, wenn mich nur jemand grundlos beleidigt und [...] mich runtermachen möchte“ (J05). Auch bei beobachteter Hatespeech kann das Positionieren eine Möglichkeit sein: ,,...] ich finde es wichtig, einfach sich genau zu positionieren, zu sagen, finde ich jetzt nicht so" (J10). Einen Schritt weiter geht das persönliche Konfrontieren der Person, die Hatespeech äußert, z.B.: ,,...] irgendwann habe ich dann auch die Nase voll und geh dann meistens auch zu den Personen hin und frag sie, ey, wenn du anderen erzählst wie doof ich bin, warum erzählst du mir das nicht?“ (J02). Diese Strategie kann ebenfalls öffentlich und mit Botschaftscharakter geäußert werden, z.B. eine Unterlassensaufforderung beinhalten oder die moralische Bewertung von Hatespeech betreffen: „Wenn es mich juckt, dann komme ich zu dem und sage dem halt einfach [...] meine Meinung. [...] Dass das nicht geht“" (J06). Im Gegensatz zum Positionieren, bei der der Fokus auf der Öffentlichkeit liegt, liegt hier der Fokus auf der Person, die Hatespeech äußert. Das Entlarven der Hatespeech kann auch Teil von Counterspeech sein und tritt im Zusammenhang mit Falschaussagen und Verallgemeinerungen auf. Hatespeech wird auf einer sachlichen Ebene öffentlich widerlegt und soll ebenjene Öffentlichkeit davon überzeugen, dass Hatespeech falsch ist: „Einfach mehrere Menschen darauf aufmerksam machen, dass das, was er tut, nicht richtig ist. [...] Bis sie [...] ihn dann konfrontieren, dass er was Falsches macht und damit aufhören sollte. Aber nicht [...] [mit] Hatespeech auf ihn, sondern mit guten Argumenten ihm zu sagen, dass es nicht richtig ist" (J19). In den Interviews zeigt sich diese Form der Counterspeech eher bei medial vermittelter Hatespeech. Eine Bewältigung durch das Ironisieren der Hatespeech kann z.B. durch schlagfertige Antworten oder durch verbale Entgegnungen geschehen, die die Aussage ins Lächerliche ziehen und so die Gesprächssituation versuchen zu lockern, z. B.: „Ich mach mich mehr darüber lustig“ (J02) oder: „Wenn mir sowas persönlich gesagt wird, [...] dann reagiere ich meistens auch mit Satire“ (J02). Es kann trotz des ironischen Charakters mit einer sachlichen Falsifikation oder einem moralischen Urteil verbunden sein und genutzt werden, um öffentlich ein Statement zu platzieren: ,,...] eigentlich würde ich am liebsten mit Satire oder mit Ironie reagieren, also einfach [...] denen ihre Grundlage so zerschmettern, dass sie [...] auch nichts mehr dagegen machen können, aber dafür muss man halt in einigen Fällen sehr schlagfertig sein“ (J05). Schlagfertigkeit wird hier als notwendige personale Ressource angeführt, um die Strategie nutzen zu können. Das Argumentieren oder Verhandeln zielt darauf ab, die Person, die Hatespeech äußert, davon zu überzeugen, dass die Aussagen falsch sind. Im Gegensatz zur Konfrontation geht es hierbei nicht nur um das Mitteilen der eigenen Botschaft, sondern um Erklärung: „Das mit der dunkelhäutigen Schülerin, dass, als ich sie einmal gefragt habe, ob sie Hautfarbe 
hat, hat sie sich darüber aufgeregt, dass Hautfarbe Hautfarbe heißt, weil schwarz ja auch eine Hautfarbe ist.“ (J04). Bei Konflikten, in denen Hatespeech genutzt wurde, kann es primär um Ausgleich gehen: ,[...] ich versuche halt immer, wenn ich so ein Problem mit jemandem habe, gehe ich halt hin und versuche es zu klären, anstatt halt dann so etwas anzufangen“ (J12). Eine weitere Möglichkeit ist gezieltes Nachfragen. Ziel ist es, die Person, die Hatespeech äußert, zu der Erkenntnis zu führen, dass dies falsch ist, z. B.: „Dass die Leute einfach nachdenken sollen. [...] Denkt darüber nach, was ihr macht und wieso ihr das macht. Denkt darüber nach, was das für Konsequenzen für andere hat" (J19). Dabei soll die Person zur Reflexion des eigenen Handelns geführt werden: ,[...] wenn es verbal passiert, finde ich, kann man/oder kann ich sagen, dass ich versuche eigentlich immer, was dazu zu sagen. Also zu sagen, wieso hast du es jetzt gesagt? Oder zu fragen, was, was er jetzt damit erreichen möchte“ (J10).

Counterspeech beschreibt Bewältigungsstrategien, bei der Hatespeech als problematisches Ereignis wahrgenommen wird. Zu vermuten ist, dass Personen, die Counterspeech nutzen, eine hohe Einfluss- bzw. Kontrollüberzeugung haben und über ausreichende personale und soziale Ressourcen verfügen, um mit aktiven Handlungen $\mathrm{zu}$ reagieren, die Hatespeech entgegenwirken sollen.

Eher gegensätzlich dazu steht das aktive Ignorieren als zweite problemfokussierte Bewältigungsstrategie, z. B.: „Ich habe die Leute einfach ignoriert, weil ich versuche [...] drüber zu stehen“ (J10). Das aktive Ignorieren wird mit dem Ziel durchgeführt, Personen, die Hatespeech äußern, bewusst nicht zu beachten und für diese uninteressant zu werden, indem potenziell interessante Reaktionen abgestellt werden: „Und [...] wenn die Leute merken, sie reagiert nicht drauf oder das macht nichts mit ihr, dann wird das denen irgendwann langweilig“ (J18). Wie in der Äußerung deutlich wird, kann das Ignorieren mit der Annahme verbunden sein, dass die zuvor gezeigte Reaktion als mögliche Ursache für die Viktimisierung durch Hatespeech attribuiert wird. Mit dem aktiven Ignorieren soll dieser Kreislauf durchbrochen werden.

Die Interpretation der Daten ergab zudem, dass die Wahl der Bewältigungsstrategie durch das subjektiv wahrgenommene Maß sozialen Rückhalts in der Klasse beeinflusst wird. Wird die soziale Unterstützung hoch eingeschätzt, tendieren Jugendliche eher zu problemfokussierten Strategien, wohingegen das Fehlen sozialer Unterstützung gegenteilig dazu führen kann, dass eher emotionsfokussierte Bewältigungsstrategien gewählt werden.

\subsection{Neubewertung}

Eine Neubewertung kann im Verlauf der Auseinandersetzung mit Hatespeech vorgenommen werden, z. B. wenn sich situative Faktoren oder Bewältigungspotenziale verändern. Neubewertung meint also eine zeitlich versetzte Wiederholung der bisherigen Bewertung. Daraus können sich Konsequenzen für die weitere oder zukünftige Bewältigung ergeben. Emotions- und problemfokussierte Strategien können dadurch auch miteinander oder aufeinander aufbauend auftreten. Berichtete Neubewertungen lassen sich finden, wenn Schüler/innen z. B. retrospektiv das eigene Handeln in einer konkreten Situation reflektieren oder Schlüsse für das zukünftige Vorgehen ziehen. 
Ist die Bewertung von negativen Emotionen bestimmt, scheinen weniger konstruktive Bewältigungsstrategien daran anzuschließen. Solche Zusammenhänge deuten sich zwischen einer von Angst bestimmten Bewertung und vermeidenden Strategien an. Wenn die Bewertung von Wut bestimmt ist, scheinen teils reaktive Aggressionen anzuschließen. Einige Interviewte reflektierten solche Zusammenhänge und zeigten sich bemüht, die so identifizierten Bewältigungspotenziale im Umgang mit Hatespeech zu bergen und eine Neubewertung aktiv zu ermöglichen. Im Datenmaterial finden sich z. B. Hinweise darauf, dass ihnen die Relevanz der Kontroll- bzw. Einflussüberzeugung für eine konstruktive Bewältigung bewusst ist, was sich im Bestreben nach Stärkung des Selbstbewusstseins (J18) oder der Bewusstmachung der Selbstwirksamkeit (J02) zeigt. Als Hypothese lässt sich dies so formulieren: Emotionsfokussierte Bewältigungsstrategien können zunächst zur gezielten Reduktion des Belastungsempfindens bzw. zur gezielten Stärkung eigener Ressourcen eingesetzt werden, um anschließend in ähnlichen Situationen mit konstruktiveren Bewältigungsstrategien reagieren zu können. Das Datenmaterial enthält Hinweise, die diese Hypothese stützen: „Aber jetzt geht mich nicht mehr so schnell an mich ran. Ich glaube, dass je mehr passiert, kannst du es mehr abweisen [...]“ (J04), sowie: „Und nach einem halben Jahr hat das dann irgendwann aufgehört, weil ich mich dann nicht mehr von den provozieren lassen habe“ (J15). Relevant erscheint das insbesondere dann, wenn Hatespeech mehrfach bzw. wiederholt erlebt wird, jedoch ein Angriff auf das Selbstbewusstsein emotionsfokussiert abgewehrt werden kann. „Und wenn ich den Schulhof betrete, durch meine kurzen Haare, durch meine Erscheinung einfach, durch mein Selbstbewusstsein, kommen Leute auf mich zu: ,Du Lesbe. ' Und das sind halt so unnötige Sachen [...]. Also das macht mit mir nichts mehr, da ich das gewohnt war früher. Da ich gemerkt habe, ich muss mein Selbstbewusstsein stärken“ (J18). Dieser Fall verdeutlicht mögliche resilienzsteigernde Effekte aufgrund der Neubewertung.

Neubewertungen zeigten sich auch, wenn sich die Hatespeech gegen veränderbare Merkmale richtete, z.B. wenn Hatespeech aufgrund von Übergewicht oder Sprachdefiziten geäußert wurde, etwa: ,Aber in der Sechsten war ich noch schwer mit Deutsch und dann so: ,Ja du Ausländer, was suchst du hier? [...] Red mal deutsch.' [...]. Also ich fand [...] die haben Recht [...]. Ich habe mir Mühe gegeben und die haben mich dann mehr runter gemacht, als mir zu helfen [...]“ (J08). Hier wird ein Merkmal angegriffen, das veränderbar erscheint (Sprachdefizit). Die Neubewertung zeigt sich darin, dass die betroffene Person der defizitären Beurteilung des Merkmals zustimmt. Es kann von einer sachlichen Umdeutung der Hatespeech oder von Assimilation gesprochen werden, z.B.: ,wenn sie [...] gesagt haben: ,ja, du bist fett", dann habe ich gesagt: ,okay dann bin ich fett"“ (J01), wobei jedoch die Gefahr einer Internalisierung der negativen Zuschreibungen bzw. einer Selbststigmatisierung gegeben ist.

\section{Diskussion}

Die vorliegende Studie ging zunächst der Frage nach, wie Hatespeech-Erfahrungen durch Schüler/innen gemäß des Transaktionalen Stressmodells (Lazarus und 
Folkman 1984) bewertet und die eigenen Bewältigungspotenziale eingeschätzt werden. Dieses hebt die Bedeutsamkeit der kognitiven Bewertung des Stressors für die Bewältigung hervor. Wie sich im Datenmaterial zeigte, wurde Hatespeech in der primären Bewertung (Lazarus und Folkman 1984) als potenziell gefährlich bzw. weiterhin als Herausforderung oder Bedrohung wahrgenommen, was sich auch an damit verbundenen, negativen Emotionen zeigte. Dieser Befund stimmt mit anderer Forschung überein, die z.B. Zusammenhänge zwischen Hatespeech-Erfahrungen und negativen Emotionen aufzeigte (Chavez et al. 2019; Eisenberg et al. 2003; Saha et al. 2019). Die sekundäre Bewertung war einerseits durch personale Ressourcen bedingt, wie z. B. der Einfluss- bzw. Kontrollüberzeugung. Andererseits fiel dabei, gerade im Setting der Schulklasse, der sozialen Unterstützung eine besondere Relevanz zu. Dieser Umstand wurde auch schon von Frydenberg (2008) erkannt: $90 \%$ der befragten Jugendlichen gaben als wichtige Ressource für die Stressbewältigung Freund/innen (bzw. mindestens ein/e nahestehende/r Freund/in) an. Die Bewältigungspotenziale wurden in den Interviews unterschiedlich hoch bzw. gering eingeschätzt. Die Berichte der Schüler/innen ließen erkennen, dass ein komplexes Zusammenspiel von sozialen Beziehungen und dem Gruppenstatus sowohl die Arten von HatespeechEreignissen als auch deren Bewältigung beeinflusst. Um Teile dieses komplexen Zusammenspiels zu erklären, lassen sich Erkenntnisse aus der Mobbingforschung entlehnen. Dort zeigte sich z. B., dass verteidigende Schüler/innen ein hohes soziales Ansehen in der Klasse und eine hohe Selbstwirksamkeitserwartung haben (Gini et al. 2008). Angst hingegen hemmte verteidigendes Verhalten bzw. Zivilcourage. Zudem konnten bereits Hinweise gefunden werden, die darauf hindeuten, dass soziale Unterstützung Diskriminierung und aggressive Verhaltensweisen verringert (Wright und Wachs 2019).

Die hinsichtlich der zweiten Forschungsfrage berichteten Bewältigungsstrategien unterschieden sich bezüglich verschiedener Situationsmerkmale, z. B. der Beeinflussbarkeit des Stressors, der Vorhersagbarkeit bzw. Unsicherheit in der Situation, der Wiederholungswahrscheinlichkeit sowie der zeitlichen Nähe und Dauer des Stressereignisses (Eschenbeck 2010; Lazarus und Folkman 1984). Der Einbezug sozialer Unterstützung ließ sich, neben Verdrängung und Vermeidung, als zentrale emotionsfokussierte Bewältigungsstrategie identifizieren. Soziale Unterstützung, als sehr breites Konstrukt, kann aber auch bei problemfokussierter Bewältigung einbezogen werden und als Ressource gelten. Aktives Ignorieren und Counterspeech ließen sich als relevante problemfokussierte Bewältigungsstrategien finden. Counterspeech konnte differenziert werden in Positionieren, Konfrontieren, Entlarven, Ironisieren, Argumentieren/Verhandeln und gezieltes Nachfragen. Es zeigte sich, dass sich die Bewältigungsstrategien in Erscheinung und Ausführung teils von denen unterscheiden, die Wachs et al. (2020b) für Online-Hatespeech finden konnten. Das ist einerseits dadurch bedingt, dass technische Lösungen (z. B. das Blockieren) nicht möglich waren, andererseits dadurch beeinflusst, dass sich Art und Umfang der Kommunikation in beiden Settings unterschieden. Im Datenmaterial deuteten sich hemmende Effekte für Counterspeech in der Schule durch die unmittelbare persönliche Konfrontation an. Gleiches könnte mutmaßlich auch für Hatespeech selbst gelten, da online soziale Mechanismen (z.B. Augenkontakt) fehlen, die Hatespeech hemmen könnten (Lapidot-Lefler und Barak 2012). Garland et al. (2020) konnten 
zeigen, dass Online-Hatespeech durch organisierte Counterspeech eingedämmt werden kann. Soziale Normen der Schulklasse bzw. Peergroup, die das soziale Handeln des Individuums normieren können, könnten in ähnlicher Weise bedeutsam sein. Die Eltern schienen bei schulischer Hatespeech weniger einbezogen zu werden als bei Online-Hatespeech (Wachs et al. 2020b). Gründe dafür könnten in der Verfügbarkeit sowie in der sozialen Dynamik der Schulklasse bzw. Peergroup liegen. Bedeutsam war online wie offline die Relevanz von sozialer Unterstützung durch Freundschaften. Gleichzeitig traten in beiden Studien die als problematisch zu bewertenden Bewältigungsstrategien Hilflosigkeit und Rache auf. In der Schule schienen darüber hinaus auch affektive, physisch gewaltvolle Handlungen hinzuzukommen.

Hinsichtlich der Neubewertung konnte auf Grundlage der Schüler/innen-Berichte ein positiver Zusammenhang zwischen der Kontroll- und Einflussüberzeugung und problemfokussierten Bewältigungsstrategien interpretiert werden, der den Interviewten teils bewusst war. Emotionsfokussierte Strategien wurden mutmaßlich angewendet, um die Selbstwirksamkeitsüberzeugung im Sinne eines Empowerments zu erhöhen und darauf aufbauend in ähnlichen Situationen mit problemfokussierten Bewältigungsstrategien reagieren zu können. Im Datenmaterial zeigten sich bei veränderbaren zugeschriebenen Merkmalen zudem Hinweise auf Anpassungsbereitschaften, die mit den von Goffman ([1963] 2012) als assimilative Techniken beschriebenen Bewältigungsstrategien übereinstimmen können.

Es ist anzunehmen, dass Defizite in den eigenen Bewältigungspotenzialen teilweise erkannt werden und versucht wird, diese auszugleichen. Ausschlaggebend sind dafür die Ressourcen: So können neue Ressourcen entdeckt oder bewusst gestärkt werden. Als Konsequenz können Veränderungen in der Wahl und Umsetzung der Bewältigungsstrategien auftreten. Erfolgreiche Bemühungen um eine Stärkung des Selbstbewusstseins bzw. der Einfluss- und Kontrollüberzeugung könnten z.B. zu einer Hinwendung zu konstruktiveren Strategien, wie Counterspeech, führen.

\subsection{Limitationen und Forschungsdesiderata}

Die vorliegende Studie trägt dazu bei, Hatespeech im Schulkontext besser zu verstehen, es muss jedoch auf gewisse methodische Einschränkungen, die die Aussagekraft begrenzen können, hingewiesen werden. Eine Limitation ergibt sich aus den landesbehördlichen Genehmigungsverfahren, da eine strenge methodische Orientierung an der Grounded Theory mit theoretischem Sampling nicht möglich war. Ein teilweiser Ausgleich erfolgte durch gezielte Kontrastierung des Samplings (purposeful Sampling) und durch die relativ große Anzahl der Interviews, von denen so nachträglich eine Fallauswahl getroffen werden konnte. Eine erschöpfende qualitative Erfassung des Untersuchungsgegenstands ist dadurch allerdings nicht vollkommen gesichert. Eine weitere methodische Einschränkung ergibt sich dadurch, dass für diesen Beitrag das Transaktionale Stressmodell und entsprechende Begriffe auf das Material angewendet wurden, was sich jedoch mit der Anschlussfähigkeit an ein fundiertes theoretisches Modell sowie eine damit einhergehende Strukturierung begründen lässt. Die Erhebung fand zudem in einem begrenzten Zeitraum statt, was unter Annahme der Veränderbarkeit von Hatespeech in Abhängigkeit von aktuellen gesellschaftlichen Ereignissen reflektiert werden muss. 
Da die Bewertungsprozesse nicht beobachtbar und Berichte darüber selektiv und subjektiv sind, ist eine Messung dieser Faktoren schwierig. Anschließende qualitative Studien, die die Bewertung fokussieren, könnten weitere Erkenntnisse zutage fördern. Dabei sollten auch die Neubewertungen nach der Erprobung von Bewältigungsstrategien differenzierter untersucht werden. Schließlich sollte zukünftige Forschung untersuchen, inwiefern Werte und Motivationen der Schüler/innen die Bewältigungsstrategie beeinflussen. Andere methodische Zugänge können die hier dargestellten Ergebnisse zudem vertiefen und erweitern.

\subsection{Praktische Implikationen}

Da sich gezeigt hat, dass Hatespeech als Stressor von negativen Emotionen begleitet wird, gilt es, Hatespeech in der Schule grundsätzlich zu verringern, um Schüler/ innen vor solchen Erfahrungen zu schützen. Dies kann durch die Förderung von demokratischen und sozialen Kompetenzen aller Schulakteur/innen unterstützt werden. Gleichzeitig sollte das pädagogische Personal für Hatespeech sensibilisiert und mit Interventions- und Präventionsmöglichkeiten vertraut gemacht werden, um adäquat handeln zu können. Da die Ergebnisse zudem gezeigt haben, dass die Einfluss- und Kontrollüberzeugung eine zentrale personale Ressource ist, um konstruktiv (z.B. mittels Counterspeech) auf Hatespeech zu reagieren, sollte diese in der Schule gestärkt werden. Hatespeech kann im Unterricht thematisiert und geeignete Gegenstrategien können besprochen und erlernt werden. Für die Herausbildung von Einfluss- und Kontrollüberzeugungen kann auch auf die Resilienzforschung verwiesen werden (Ungar et al. 2013). Eine ressourcenorientierte Herangehensweise im Schulalltag kann hierzu förderlich sein. Weiterhin kann konstruktive Bewältigung durch soziale Unterstützung begünstigt werden, sodass Prävention konkret durch die Entwicklung eines sozial unterstützenden Umfelds erfolgen kann. Die Stärkung von sozialen Beziehungen und verbindliche Normen sowie gemeinsame Grundwerte auf Schul- und Klassenebene sind bereits aus der Mobbingforschung als präventive Möglichkeiten bekannt (Schubarth et al. 2017; Schubarth 2019) und können auf Hatespeech übertragen werden. Die partizipative Einbindung der Schüler/innen ist dabei zu beachten.

\subsection{Konklusion}

Ziel der Studie war es, herauszufinden wie von Hatespeech betroffene Schüler/ innen diese bewerten und bewältigen sowie dies unter Anwendung des Transaktionalen Stressmodells (Lazarus und Folkman 1984) abzugleichen. Insgesamt bleibt festzuhalten, dass sich das Modell gut auf die Studienergebnisse anwenden lässt, in denen sich Belege für primäre und sekundäre Bewertung sowie Neubewertung und verschiedene emotions- und problemfokussierte Bewältigungsstrategien finden lassen. Als besonders konstruktive Bewältigung erscheinen dabei die identifizierten Formen der Counterspeech. Weiterer Forschungsbedarf besteht insbesondere zu der Frage, wie Lehrkräfte mit Hatespeech umgehen und welche Strategien hierbei erfolgversprechend sind. Die Schüler/innen sollten darin unterstützt werden, konstruktive Bewältigungsstrategien im Umgang mit Hatespeech zu finden und eine 
resiliente Persönlichkeit zu entwickeln. Die hier gelieferten Erkenntnisse können zur Entwicklung von Präventionsprogrammen genutzt werden und dabei helfen, die Schule als sozialen Lernort weiterzuentwickeln. Schüler/innen sollten dabei stets partizipativ als aktiv handelnde Expert/innen ihrer Lebenswelt in die Bewältigung dieser Herausforderung eingebunden werden.

Förderung Die vorliegende Studie entstand im Rahmen eines Kooperationsprojekts zwischen der Universität Potsdam (Dr. Sebastian Wachs, Prof. Dr. Wilfried Schubarth) und der Brandenburgischen Technischen Universität Cottbus-Senftenberg (Prof. Dr. Ludwig Bilz). Das Forschungsprojekt „Hate Speech als Schulproblem" wurde mit Mitteln der Deutschen Forschungsgemeinschaft unterstützt (Geschäftszeichen: WA 4275/2-1; BI 1046/9-1; SCHU 1370/4-1).

Funding Open Access funding enabled and organized by Projekt DEAL.

Open Access Dieser Artikel wird unter der Creative Commons Namensnennung 4.0 International Lizenz veröffentlicht, welche die Nutzung, Vervielfältigung, Bearbeitung, Verbreitung und Wiedergabe in jeglichem Medium und Format erlaubt, sofern Sie den/die ursprünglichen Autor(en) und die Quelle ordnungsgemäß nennen, einen Link zur Creative Commons Lizenz beifügen und angeben, ob Änderungen vorgenommen wurden.

Die in diesem Artikel enthaltenen Bilder und sonstiges Drittmaterial unterliegen ebenfalls der genannten Creative Commons Lizenz, sofern sich aus der Abbildungslegende nichts anderes ergibt. Sofern das betreffende Material nicht unter der genannten Creative Commons Lizenz steht und die betreffende Handlung nicht nach gesetzlichen Vorschriften erlaubt ist, ist für die oben aufgeführten Weiterverwendungen des Materials die Einwilligung des jeweiligen Rechteinhabers einzuholen.

Weitere Details zur Lizenz entnehmen Sie bitte der Lizenzinformation auf http://creativecommons.org/ licenses/by/4.0/deed.de.

\section{Literatur}

Chavez, L.R., Campos, B., Corona, K., Sanchez, D., \& Ruiz, C. B. (2019). Words hurt: political rhetoric, emotions/affect, and psychological well-being among Mexican-origin youth. Social Science \& Medicine, 228, 240-251. https://doi.org/10.1016/j.socscimed.2019.03.008.

Compas, B.E., Connor-Smith, J. K., Saltzman, H., Thomsen, A. H., \& Wadsworth, M.E. (2001). Coping with stress during childhood and adolescence: Problems, progress, and potential in theory and research. Psychological Bulletin, 127(1), 87-127. https://doi.org/10.1037/0033-2909.127.1.87.

Denzin, N. K. (1989). The research act: a theoretical introduction to sociological methods. : Prentice Hall.

Dresing, T., \& Pehl, T. (2018). Praxisbuch Transkription: Regelsysteme, Software und praktische Anleitungen für qualitative ForscherInnen (8. Aufl.).

Eisenberg, M.E., Neumark-Sztainer, D., \& Story, M. (2003). Associations of weight-based teasing and emotional well-being among adolescents. Archives of pediatrics \& adolescent medicine, 157(8), 733-738. https://doi.org/10.1001/archpedi.157.8.733.

Eschenbeck, H. (2010). Bewältigung alltäglicher Stresssituationen von Kindern und Jugendlichen. Zeitschrift für Gesundheitspsychologie, 18(3), 103-118. https://doi.org/10.1026/0943-8149/a000018.

Flick, U. (2014). Gütekriterien qualitativer Sozialforschung. In N. Baur \& J. Blasius (Hrsg.), Handbuch Methoden der empirischen Sozialforschung (S. 411-423). Wiesbaden: Springer.

Flick, U. (2019). Qualitative Sozialforschung: Eine Einführung (9. Aufl.). Rororo Rowohlts Enzyklopädie, Bd. 55694. Hamburg: Rowohlt.

Frydenberg, E. (2008). Adolescent coping: advances in theory, research and practice. : Routledge. https:// doi.org/10.4324/9780203938706.

Garland, J., Ghazi-Zahedi, K., Young, J.-G., Hébert-Dufresne, L., \& Galesic, M. (2020). Impact and dynamics of hate and counter speech online. http://arxiv.org/pdf/2009.08392v2. Zugegriffen: 10.02.2021.

Gini, G., Albiero, P., Benelli, B., \& Altoè, G. (2008). Determinants of adolescents' active defending and passive bystanding behavior in bullying. Journal of adolescence, 31(1), 93-105. https://doi.org/10. 1016/j.adolescence.2007.05.002. 
Glaser, B. G., \& Strauss, A. L. (1998). Grounded theory. : Huber.

Goffman, E. (2012). Stigma: Über Techniken der Bewältigung beschädigter Identität (21. Aufl.). Suhrkamp-Taschenbuch Wissenschaft, Bd. 140. : Suhrkamp.

Gámez-Guadix, M., Wachs, S., \& Wright, M. F. (2020). "Haters back off!”. Psychometric properties of the coping with Cyberhate questionnaire and relationship with well-being among Spanish adolescents. Psicothema, 32(4), 567-574.

Hansen, N. (2009). Die Verarbeitung von Diskriminierung. In A. Beelmann \& K. J. Jonas (Hrsg.), Diskriminierung und Toleranz (S. 155-170). : VS. https://doi.org/10.1007/978-3-531-91621-7_8.

Hawdon, J., Bernatzky, C., \& Costello, M. (2019). Cyber-routines, political attitudes, and exposure to violence-advocating online extremism. Social Forces, 98(1), 329-354. https://doi.org/10.1093/sf/ soy 115 .

Helfferich, C. (2014). Leitfaden- und Experteninterviews. In N. Baur \& J. Blasius (Hrsg.), Handbuch Methoden der empirischen Sozialforschung (S. 559-574). Wiesbaden: Springer.

Hunter, S.C., \& Boyle, J. M.E. (2004). Coping and appraisal in victims of school bullying. British Journal of Educational Psychology, 74(1), 83-107.

Lapidot-Lefler, N., \& Barak, A. (2012). Effects of anonymity, invisibility, and lack of eye-contact on toxic online disinhibition. Computers in Human Behavior, 28(2), 434-443. https://doi.org/10.1016/j.chb. 2011.10.014.

Lazarus, R. S., \& Folkman, S. (1984). Stress, appraisal, and coping. Berlin Heidelberg: Springer.

Mayring, P. (2015). Qualitative Inhaltsanalyse: Grundlagen und Techniken (12. Aufl.). : Beltz.

Palinkas, L. A., Horwitz, S. M., Green, C. A., Wisdom, J. P., Duan, N., \& Hoagwood, K. (2015). Purposeful sampling for qualitative data collection and analysis in mixed method implementation research. $A d$ ministration and policy in mental health, 42(5), 533-544. https://doi.org/10.1007/s10488-013-0528$\mathrm{y}$.

Saha, K., Chandrasekharan, E., \& de Choudhury, M. (2019). Prevalence and psychological effects of hateful speech in online college communities. In P. Boldi, B. F. Welles, K. Kinder-Kurlanda, C. Wilson, I. Peters \& W. Meira (Hrsg.), Proceedings of the 10th ACM Conference on Web Science-WebSci '19 (S. 255-264). : ACM Press. https://doi.org/10.1145/3292522.3326032.

Scherr, A., \& Breit, H. (2018). Erfolgreiche Bewältigung von Diskriminierung. In P. Genkova \& A. Riecken (Hrsg.), Handbuch Migration und Erfolg. Psychologische und sozialwissenschaftliche Aspekte. Springer Reference Psychologie. (S. 1-24). Wiesbaden: Springer.

Schubarth, W. (2019). Gewalt und Mobbing an Schulen: Möglichkeiten der Prävention und Intervention. Bd. 3. Stuttgart: Kohlhammer.

Schubarth, W., Ulbricht, J., \& Bilz, L. (2017). Lehrerhandeln bei Gewalt und Mobbing: Zentrale Ergebnisse einer Studie. Pädagogik, 69(5), 40-43.

Soral, W., Bilewicz, M., \& Winiewski, M. (2018). Exposure to hate speech increases prejudice through desensitization. Aggressive behavior, 44(2), 136-146. https://doi.org/10.1002/ab.21737.

Strauss, A., \& Corbin, J. (2010). Grounded theory: Grundlagen qualitativer Sozialforschung. : Beltz.

Strübing, J. (2014). Grounded Theory: Zur sozialtheoretischen und epistemologischen Fundierung eines pragmatistischen Forschungsstils (3. Aufl.). Wiesbaden: VS.

Tynes, B. M., Giang, M.T., Williams, D. R., \& Thompson, G. N. (2008). Online racial discrimination and psychological adjustment among adolescents. The Journal of Adolescent Health: Official Publication of the Society for Adolescent Medicine, 43(6), 565-569. https://doi.org/10.1016/j.jadohealth.2008.08. 021.

UK Safer Internet Centre. (2016). Creating a better internet for all: young people's experiences of online empowerment + Online hate. http://childnetsic.s3.amazonaws.com/ufiles/SID2016/Creating\%20a \%20Better\%20Internet\%20for\%20All.pdf. Zugegriffen: 10.02.2021.

Ungar, M., Bottrell, D., Tian, G.-X., Wang, X., Whittaker-Stemmler, J., Pfeiffer, J., \& Stemmler, G. (2013). Resilienz: Stärken und Ressourcen im Jugendalter. In C. Steinebach \& K. Gharabaghi (Hrsg.), Resilienzförderung Im Jugendalter: Praxis und Perspektiven (S. 1-19). Berlin Heidelberg: Springer. https:// doi.org/10.1007/978-3-642-33048-3_1.

Wachs, S., Schubarth, W., \& Bilz, L. (2020a). Hate Speech als Schulproblem? In I. van Ackeren, H. Bremer, F. Kessl, H. C. Koller, N. Pfaff, C. Rotter, D. Klein \& U. Salaschek (Hrsg.), Bewegungen: Beiträge zum 26. Kongress der Deutschen Gesellschaft für Erziehungswissenschaft (S. 223-236). : Barbara Budrich. https://doi.org/10.2307/j.ctv10h9fjc.19.

Wachs, S., Ballaschk, C., Krause, N., \& Schubarth, W. (2020b). Bewältigung von Hate Speech im Jugendalter. Welche Rolle spielt die elterliche Medienerziehung? Zeitschrift für Jugendkriminalrecht und Jugendhilfe, 2, 131-138. 
Wachs, S., Ballaschk, C., Krause, N., Schubarth, W., Schulze-Reichelt, F., \& Bilz, L. (im Druck) Hate Speech als Herausforderung für Schule und Lehrkräftebildung. In S. Wachs, B. Koch-Priewe \& A. Zick (Hrsg.), Hate Speech-Multidisziplinäre Analysen und Handlungsoptionen. Wiesbaden: VS.

Wright, M.F., \& Wachs, S. (2019). Does social support moderate the relationship between racial discrimination and aggression among Latinx adolescents? A longitudinal study. Journal of adolescence, 73, 85-94. https://doi.org/10.1016/j.adolescence.2019.04.001.

Wright, M.F., Wachs, S., \& Gámez-Guadix, M. (2021). Youths' coping with cyberhate: Roles of parental mediation and family support. Comunicar. https://doi.org/10.3916/C67-2021-02. 